\title{
Pessoas muito inteligentes são uma ameaça
}

\author{
Very smart people are a threat
}

DOI: $10.46932 / s f j d v 2 n 4-071$

Received in: March 1st, 2021

Accepted in: May 30th, 2021

\section{Fabiano de Abreu Rodrigues}

$\mathrm{PhD}$, neurocientista, mestre psicanalista, biólogo, historiador, antropólogo, com formações também em neuropsicologia, psicologia, neurolinguística, neuroplasticidade, inteligência artificial, neurociência aplicada à aprendizagem, filosofia, jornalismo e formação profissional em nutrição clínica - Diretor do Centro de Pesquisas e Análises Heráclito; Chefe do Departamento de Ciências da Logos University;

Membro da Federação Européia de Neurociências e da Sociedade Brasileira e Portuguesa de Neurociências. Universidades em destaque: Logos University, Nova de Lisboa, Faveni, edX Harvard, Universidad de Madrid.

\section{RESUMO}

A inteligência é quando o indivíduo possui capacidades como lógica, abstração, memorização, compreensão, autoconhecimento, comunicação, aprendizado, controle emocional, planejamento e resolução de problemas. O objetivo do presente estudo é compreender o porquê de as pessoas inteligentes serem consideradas uma ameaça. O presente estudo trata-se de uma revisão bibliográfica desenvolvida em nas bases de dados PubMed e Scielo e Cinahl. Devido aos seus níveis de conhecimento em diversas áreas, possibilitando que consigam argumentar em qualquer situação ou com qualquer pessoa.

Palavras-chave: inteligência, neurociência, cérebro, cortéx pré-frontal, argumentos, persuasão

\section{ABSTRACT}

Intelligence is when the individual has resources such as; logic, abstraction, memorization, understanding, self-knowledge, communication, learning, emotional control, planning and problem solving. The aim of this study is to understand why smart people are considered a threat. The present study is a literature review developed in PubMed and Scielo and Cinahl databases. Learning at their knowledge levels in different areas, enabling them to be able to argue in any situation or with anyone.

Keywords: intelligence, neuroscience, brain, prefrontal cortex, arguments, persuasion

\section{INTRODUÇÃO}

\subsection{INTELIGÊNCIA}

O termo surgiu do latim intelligentīa. A origem etimológica é referente a quem sabe escolher, permitir, então, que o indivíduo escolha melhores opções na hora de solucionar alguma questão (Hajisabbagh, 2020).

Seu conceito foi estudado pela primeira vez com o psicólogo inglês chamado Charles Spearmen, no início do século XX. Spearman cunhou o termo 'Inteligência Geral' ou 'g', que baseou-se na medida do desempenho das pessoas em uma variedade de testes mentais (Hajisabbagh, 2020). 
A psicologia acredita em dois tipos de inteligência que, juntas, constituem o g de Spearman: inteligência cristalizada e fluida. A primeira está associada ao conhecimento e experiência anteriores e reflete a cognição verbal. Já a inteligência fluida requer raciocínio adaptativo em situações. A inteligência cristalizada é caracterizada pela habilidade verbal, influenciada pela estrutura e espessura cortical em áreas laterais dos lobos temporais. Enquanto as áreas parietais expõem a sobreposição de seu envolvimento na inteligência cristalizada e demais tipos de inteligência. A área temporal de Brodman 38 está envolvida somente na inteligência cristalizada (Gainotti, 2006; Rodrigues1, 2021).

$\mathrm{O}$ córtex frontal lateral está relacionado ao raciocínio, atenção, memória, sendo relacionada à inteligência fluida, bem como o lobo parietal. Estudos sobre inteligência fluida usando Matrizes Progressivas Avançadas de Raven comprovaram a ativação de diversas áreas no hemisfério esquerdo, em particular o córtex posterior. Pessoas que apresentam uma inteligência fluídica maior possuem atividade neural nas regiões pré-frontal e parietal lateral (Gray, 2005).

Para Webb et al. 2014, as medidas do volume da substância cinzenta de duas áreas frontais, órbitofrontal (OFC) e córtices cingulados anteriores rostrais (rACC), foram complementadas pela conectividade da substância branca entre essas regiões. Junto a isso, o volume da substância cinzenta esquerda e da conectividade da substância branca entre a órbito-frontal posterior esquerdo e o rACC demonstraram que até $50 \%$ da variância na inteligência geral principalmente no córtex pré-frontal em relação à sua estrutura, função e conectividade estão relacionadas à inteligência geral, especificamente à capacidade de raciocínio e à memória.

\subsection{QUOCIENTE DE INTELIGÊNCIA (Q.I.)}

O QI é uma escala que auxilia na avaliação e comparação, das habilidades de diversos indivíduos nas áreas do pensamento, como matemática básica, raciocínio ou lógica, por exemplo. Tal valor é gerado por meio da realização de testes que avaliam uma ou várias áreas. Isso significa que o valor do QI deve ser usado apenas para avaliar as mesmas áreas do conhecimento (Mohamed, 2020; Rodrigues, 2021).

Sendo a principal vantagem a de o indivíduo aprender coisas novas ou para aprimorar uma determinada função. Indivíduos com um QI mais alto na maioria das vezes precisam de menos informação para aprender algo novo, ou são mais adequados para desempenhar uma função, enquanto pessoas com um QI menor precisam de mais tempo e de informações mais detalhadas (Gardner, 2020).

As classificações de QI são: superior a 140 significa inteligência genial; entre 120 e 140 representa inteligência muito superior; entre 110 e 120 indica inteligência superior; entre 90 e 110 exprime inteligência normal ou média; entre 80 a 90 manifesta torpeza, raramente qualificada como debilidade mental; entre 70 e 80 denota debilidade mental fronteiriça; abaixo de 70 revela debilidade mental definida 
(Gardner, 2020).

\subsection{NEUROCIÊNCIA E INTELIGÊNCIA}

Os lados esquerdo e direito do córtex pré-frontal ou lobo frontal são ligados por um feixe grosso de fibras nervosas chamado de corpo caloso. Tais fibras são de cor branca (conjunto de axônios e dendritos) e responsáveis pela troca de informações entre as distintas áreas do córtex cerebral (Focus, 2020).

Em mamíferos maiores (como seres humanos, primatas e cetáceos), o cérebro possui uma superfície irregular, cheia de áreas mais protuberantes (giros), intercaladas com pequenos vales (sulcos), que geram aspecto de enrugamento no cérebro. Já o cérebro de mamíferos menores, como camundongos e ratos, possuem poucos giros e sulcos, e sua superfície é mais lisa (Focus, 2020).

Existe uma variação de 6,7\% na inteligência de um indivíduo ao tamanho de seu cérebro. A frequência da atividade, no lado esquerdo do córtex pré-frontal, corresponde a uma variação de $10 \%$ ao desempenho intelectual (Focus, 2020).

Figura 1 - Córtex pré-frontal

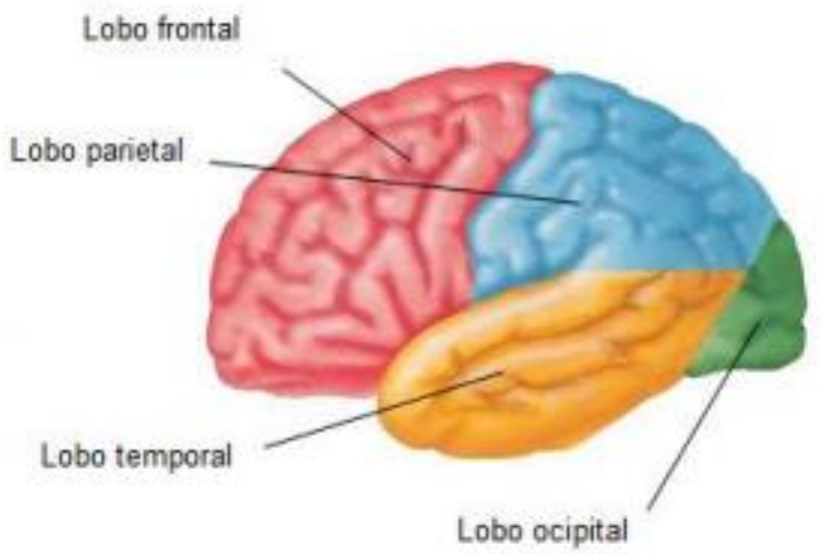

Fonte: Adaptado de Folha et. al. 2013

\section{OBJETIVO}

Compreender o porquê de as pessoas inteligentes serem consideradas uma ameaça.

\section{METODOLOGIA}

O presente estudo trata-se de uma revisão bibliográfica desenvolvida nas bases de dados PubMed e Scielo e Cinahl, utilizando os seguintes termos em português: inteligência, cérebro, córtex pré-frontal, argumentos, persuasão e em inglês: intelligence, brain, prefrontal cortex, arguments, persuasion. 


\section{RESULTADOS}

\subsection{PESSOAS INTELIGENTES E SEUS FORTES ARGUMENTOS DE CONVENCIMENTO.}

A maioria dos indivíduos inteligentes são persuasivos. Isso significa que possuem o poder de convencer outras pessoas de que o seu ponto de vista está correto. Ser persuasivo é diferente de ser manipulador, pois o primeiro apresenta fatos verídicos (Turjamaa, 2019).

O indivíduo persuasivo possui facilidade devido a sua inteligência. Consegue se comunicar de maneira mais adequada, como possui conhecimentos variados, é capaz de encontrar argumento em diversas situações.

As principais características desses indivíduos são: Otimismo, por ter ciência do próprio conhecimento, trazendo conforto e segurança aos demais. Autoconfiança, indivíduos que atuam com mais confiança possuem vantagem até nos mais competentes. Pois, para persuadir, é necessário impulsionar sua autoestima sem modificar isso em algo agressivo. Autenticidade, as pessoas não confiam em indivíduos forçados, pois assim conseguem criar uma conexão emocional. Conscientes, assim é mais fácil com que o indivíduo confie em você e seja mais fácil convencê-lo. Além disso, Apreciam o silêncio, pois, segundo pesquisadores comportamentais, indivíduos que falam muito geralmente perdem (Bergren, 2018).

Pessoas inteligentes geralmente têm formação acadêmica e muito conhecimento pela personalidade curiosa. Em alguns casos utilizam grandes teóricos para embasar seus pensamentos, lançam mão de seu arcabouço teórico para provar suas ideias, possuem repertório de fala, velocidade no processamento de dados, argumentação clara e convincente (Bergren, 2018).

Fato é que discutir ou debater com pessoas inteligentes requer criatividade a serviço da inteligência, pois há a necessidade de articular o pensamento para derivar conhecimento e assim encontrar diversidade de defesa. Nessas divergências não cabem agressões nem violência, pois não são pessoas contra pessoas e sim ideias contraponto ideias. É uma conversa mental desafiadora, e o que ocorre é que enriquece quem fala e quem escuta, e no final vence o mais inteligente (Bergren, 2018).

\subsection{PESSOAS MUITO INTELIGENTES SÃO UMA AMEAÇA}

Devido ao fato de os indivíduos muito inteligentes terem conhecimento nas mais diversas áreas, consequentemente conseguirão argumentar melhor com outro indivíduo (Majumder, 2017).

Sendo assim, quando o outro perde uma disputa, seja física ou intelectual, ocorre um momento de decepção consigo mesmo, que na maioria das vezes é levado a um processo de raiva e é descontado em cima do outro (Majumder, 2017).

O perdedor, não contente em ser vencido, pode acabar sentindo que seu oponente é uma ameaça, 
levando-o a achar que o indivíduo é arrogante e prepotente (Cho, 2020).

Um grande exemplo de líder com alto QI e que foi amado, porém muito mais odiado devido às consequências das atrocidades, considerado uma grande ameaça, foi o político alemão Adolf Hitler, líder do partido Nazista, conhecido também por seus poderes de persuasão e convencimento (Rodriguez2, 2021).

\section{CONSIDERAÇÕES FINAIS}

A partir dos resultados apresentados é possível compreender o porquê de indivíduos inteligentes serem considerados uma ameaça. Devido aos seus níveis de conhecimento em diversas áreas, possibilitando que consigam argumentar em qualquer situação, ou com qualquer pessoa. Causando aos que com ele convivem sentimentos contraditórios.

Admiração pela sua competência em envolver o ouvinte com argumentos lógicos, interessantes, sendo competentes em sua explanação e formando seguidores. Ou temor, por serem destaques sempre com sua lucidez e facilidade de liderança.

Os menos favorecidos intelectualmente sentem-se atraídos, envolvidos e seduzidos, ou sentem aversão, constrangimento, timidez por não estarem à altura de uma conversa em que possam contraargumentar de forma a fazer frente às ideias destes sujeitos.

A característica da mente brilhante gera no outro, normalmente, sentimentos opostos, atração ou aversão. Pessoas com alto QI podem ter problemas de relacionamentos por estar sempre à frente em sua leitura de cenário e ter uma interpretação mais assertiva e criativa para soluções de questões de ordem prática do cotidiano. 


\section{REFERÊNCIAS}

BERGREN, M.D. Persuasive Presentations: How to Speak So People Will Listen. NASN Sch Nurse. n. 33, v. 4, págs. 220-221, 2018 doi: 10.1177/1942602X18778238

CHO J, Lee HE. Post-adoption beliefs and continuance intention of smart device use among people with physical disabilities. Disabil Health J. 2020 Apr;13(2):100878. doi: 10.1016/j.dhjo.2019.100878. Epub 2019 Dec 5. PMID: 31859232.

Focus F.B. Neuroscience methods. Nat Neurosci. n. 23, v. 12, págs. 1455, 2020 doi: 10.1038/s41593-02000750-Z.

FOLHA, O.A.A.C., Junior, P.A. Bahia, C.P. Efeitos do ambiente sobre o período crítico de plasticidade do córtex pré-frontal de ratos adolescentes. Dissertação do PPG em neurociências e biologia celular da Universidade Federal do Pará, Belém, 2013

GAINOTTI, G. Anatomical functional and cognitive determinants of semantic memory disorders. Neurosci. Biobehav. Rev. n. 30, págs. 577-594, 2006 doi: 10.1016/j.neubiorev.2005.11.001

GARDNER, R.M., Dalman, C., Rai, D., Lee, B.K., Karlsson, H. The Association of Paternal IQ With Autism Spectrum Disorders and Its Comorbidities: A Population-Based Cohort Study. J Am Acad Child Adolesc Psychiatry. n. 59, v. 3, págs. 410-421, 2020 doi: 10.1016/j.jaac.2019.04.004.

GRAY, J.R., Chabris, C.F., e Braver, T.S. Neural mechanisms of general fluid intelligence. Nat. Neurosci. v. 6, págs. 316-322, 2003 doi: 10.1038/nn1014

HAIER, R.J., Jung, R.E., Yeo, R.A., Head, K., Alkire, M.T. The neuroanatomy of general intelligence: sex matters. Neuroimage n. 25, págs. 320-327, 2005 doi: 10.1016/j.neuroimage.2004.11.019

HAJISABBAGH, N., Fereidooni-Moghadam, M., Masoudi, R., Etemadifar, M. The effect of an emotional intelligence component program on happiness in patients with epilepsy. Epilepsy Behav. n. 106, e106972, 2020 doi: 10.1016/j.yebeh.2020.106972.

MAJUMDER S, Aghayi E, Noferesti M, Memarzadeh-Tehran H, Mondal T, Pang Z, Deen MJ. Smart Homes for Elderly Healthcare-Recent Advances and Research Challenges. Sensors (Basel). 2017 Oct 31;17(11):2496. doi: 10.3390/s17112496.

MOHAMED, I.N., Osman, A.H., Mohamed, S., Hamid, E.K., Hamed, A.A., Alsir, A., Intelligence quotient (IQ) among children with epilepsy: National epidemiological study - Sudan. Epilepsy Behav. $\mathrm{n}$. 103, e106813, 2020 doi: 10.1016/j.yebeh.2019.106813

RODRIGUES1, FA. Dwri Intelligence And Other Intelligences. International Journal of Development Research. 11(1):43576-43584 https://doi.org/10.37118/ijdr.20911.01.2021

RODRIGUES2, FA Hitler: Como um alto QIdomina e convence e como um transtorno derruba. Brazilian Journal of Development. 7(1);2021

TURJAMAA, R., Pehkonen, A., Kangasniemi, M. How smart homes are used to support older people: An integrative review. Int J Older People Nurs. n. 14, v. 4, e12260, 2019 doi: 10.1111/opn.12260. 
WEBB, C.A., Weber, M., Mundy, E.A., Killgore, W.D. Reduced gray matter volume in the anterior cingulate, orbitofrontal cortex and thalamus as a function of mild depressive symptoms: a voxelbased morphometric analysis. Psychol Med. n. 44, v. 13, págs. 2833-2843, 2014 doi: 10.1017/S0033291714000348 